\title{
PREMIERS RÉSULTATS CONCERNANT LA SUPPRESSION D'UNE OU PLUSIEURS TRAITES PAR SEMAINE CHEZ LA BREBIS SARDE
}

\author{
S. CASU et J. LABUSSIÈRE \\ Istituto Zootecnico e Caseario per la Sardegna (Bonassai-Sassari) \\ Laboratoive de la Chaire de Zootechnie, \\ Ecole nationale supérieure agronomique, \\ Centre de Recherches de Rennes, I. N. R. A., \\ 35 - Rennes

\section{RÉSUMÉ}

48 brebis Sardes sont subdivisées en 4 groupes homogènes après une période préexpérimentale de I $_{5}$ jours pendant laquelle on pratique 2 traites quotidiennes sur tous les animaux .

Les traitements appliqués ensuite à ces 4 groupes pendant I 47 jours sont les suivants :

Lot $I: 2$ traites par jour à 6 et 18 heures (témoin)

Lot 2 : I traite par jour à 6 heures

Lot $3: 2$ traites par jours du lundi au samedi à 6 et I 8 heures et une seule traite le dimanche à 6 heures

Lot 4: 2 traites par jour du lundi au samedi et suppression des 2 traites du dimanche.

Les résultats obtenus tendent à prouver que la brebis Sarde est capable de supporter de grands intervalles de traite. Ceci est probablement dû aux particularités de stockage considérable des parties citernales de la glande mammaire. Par rapport au lot $\mathbf{I}$ témoin, les productions laitières des lots 2,3 et 4 sont respectivement réduites de 5,2 p. Ioo, I2,7 p. Ioo et 23,9 p. IOO (tabl. I).

Les taux butyreux et les taux azotés moyens ne diffèrent pas significativement d'un groupe à l'autre : toutefois, les quantités totales de matières grasses et de matières azotées produites pendant les $2 \mathrm{I}$ semaines expérimentales sont sensiblement plus faibles lorsqu'on allonge l'intervalle entre traites (surtout pour le groupe 4 , tabl. I).

Comme cela avait été constaté dans l'espèce bovine, la sécrétion du lait et sa composition sont fortement perturbées jusqu'au mercredi. Dans les élevages qui adopteraient l'une de ces techniques, il faudrait donc exclure le passage du contrôleur laitier avant le jeudi (fig. 2, 3 et 4 , tabl. 2).

Aucune des 48 brebis étudiées n'a manifesté de mammites cliniques au cours de l'expérience. Sur les groupes 3 et 4 , on a toutefois pu déceler une augmentation du nombre de leucocytes du lait pendant les 4 jours qui suivent la rétention lactée (test CMT). 


\section{INTRODUCTION}

La traite biquotidienne du bétail laitier interdit à l'éleveur de s'absenter plus de quelques heures de son exploitation. Il en résulte que ce dernier accepte de moins en moins facilement la comparaison de ses conditions de vie avec celles plus favorables des autres secteurs productifs.

C'est dans le but d'humaniser ce travail de plus en plus contraignant que des essais de suppression d'une ou plusieurs traites par semaine ont été entrepris ces dernières années chez les bovins (voir revue bibliographique de LABUssik̀ RE et RICHARD, I965).

Il ressort de ces travaux :

I $^{\circ}$ Qu'il est difficilement concevable de ne traire qu'une seule fois par jour pendant toute la lactation tant que nous ne serons pas en mesure de trouver un critère simple de détection des pertes probables qui peuvent varier entre $25 \mathrm{p}$. Ioo et 89 p. IOO (CLAESSON et al., I959).

$2^{\circ}$ Qu'il est possible de ne plus traire le dimanche soir puisque CLAESSON (I962) et LABUSSIÈrE et CoINDE'T (Ig68) s'accordent pour reconnaître que l'état sanitaire du pis n'est pas affecté et que la quantité de lait produite en 300 jours est seulement réduite d'environ 5 à Io $\mathrm{p}$. Ioo.

Comme à notre connaissance il n'existe pas de travaux similaires de longue durée dans l'espèce ovine, nous avons jugé utile d'entreprendre une série de recherches pour établir les limites d'application de ces nouvelles techniques dans les conditions de l'élevage méditerranéen, compte tenu en particulier des grandes possibilités de stockage du lait citernal dans la mamelle de la brebis Sarde.

\section{MATÉRIEL E'T MÉTHODE}

L'expérience réalisée à partir de janvier 1969 à la ferme Bonassai de l'Istituto Zootecnico e Caseario per la Sardegna (Sassari) porte sur 48 brebis adultes.

\section{A. - Constitution des groupes expérimentaux}

Le sevrage intervient entre le $30^{\mathrm{e}}$ et le $44^{\mathrm{e}}$ jour après l'agnelage. On contrôle alors régulièrement pendant ${ }_{5}$ jours la production laitière obtenue aux 2 traites quotidiennes. Compte tenu des résultats, les brebis sont subdivisées en 4 groupes homogènes. Elles sont alors au $45^{\mathrm{e}}{ }_{5} 8^{\mathrm{e}}$ jour de lactation, elles resteront en expérience pendant $\mathrm{I} 47$ jours.

Groupe $1: 2$ traites par jour, à 6 heures et I 8 heures.

Groupe 2: I traite par jour, à 6 heures.

Groupe 3: 2 traites par jour du lundi au samedi à 6 heures et 18 heures et une seule traite le dimanche à 6 heures.

Groupe 4: 2 traites par jour du lundi au samedi et pas de traite le dimanche. Il s'écoule donc 36 heures entre la traite du samedi soir et celle du lundi matin.

\section{B. - Conduite du troupeau}

Toutes les brebis passent la nuit dans un enclos ouvert annexé à la bergerie. Pendant la journée, les animaux restent au pâturage (de 7 heures à $I_{7}$ heures 45 ) sur un herbage d'avoine 
et de trèfle d'Alexandrie (ou de ray-grass d'Italie et trèfle d'Alexandrie). De l'orge est distribuée chaque matin à raison de $300 \mathrm{~g} / \mathrm{j}$ et par tête.

La traite mécanique sans repasse manuelle est effectuée dans une installation à fosse, modèle Bonassai comprenant 6 faisceaux trayeurs.

\section{C. - Contrôles expérimentaux}

Les mesures individuelles de la production du lait (par pesée), du taux butyreux (méthode Gerber) et du taux de matières azotées (méthode colorimétrique à l'orange $G$ ) ont lieu chaque jour à chaque traite pendant toute la durée de l'expérience.

L'état sanitaire de la mamelle est vérifié toutes les deux semaines, du samedi matin au mercredi matin, à l'aide du California Mastitis Test (CMT) qui permet une numération sommaire des leucocytes du lait.

\section{RÉSULTATS}

\section{A. - Production laitière}

I. Bilan des 147 jours.

La production du groupe I ( 2 traites journalières) est supérieure de :

( 5,2 p. IOo à celle du groupe 2 (traite une seule fois par jour)

Tableau $I\left\{\begin{array}{l}\text { I2,7 p. Ioo à celle du groupe } 3 \text { (pas de traite le dimanche soir) } \\ \bullet 23,9 \text { p. Ioo à celle du groupe } 4 \text { (aucune traite le dimanche). }\end{array}\right.$

Le test de Duncan nous apprend que les seules différences significatives se situent entre 4 d'une part et I et 2 d'autre part.

TABL,EAU I

Effets de différentes fréquences de traite sur la production du lait et de ses principaux constituants (en 147 jours)

\begin{tabular}{|c|c|c|c|c|c|c|c|c|c|c|}
\hline \multirow{2}{*}{ Traitement } & \multicolumn{2}{|c|}{$\begin{array}{l}\underset{(\mathrm{kg})}{\mathrm{Lait}}\left({ }^{1}\right) \\
\end{array}$} & \multicolumn{2}{|c|}{$\begin{array}{l}\text { Mat. grasses }(\mathbf{1}) \\
(\mathrm{kg})\end{array}$} & \multicolumn{2}{|c|}{$\begin{array}{c}\text { Taux butyreux } \\
(\%)\end{array}$} & \multicolumn{2}{|c|}{$\begin{array}{c}\text { Mat. azotées }\left({ }^{1}\right) \\
(\mathrm{kg})\end{array}$} & \multicolumn{2}{|c|}{$\begin{array}{c}\text { Taux azoté } \\
(\%)\end{array}$} \\
\hline & $\overline{\mathrm{X}}$ & $\sigma$ & $\bar{x}$ & $\sigma$ & $\overline{\mathrm{X}}$ & $\sigma$ & $\overline{\mathrm{x}}$ & $\sigma$ & $\overline{\mathrm{x}}$ & $\sigma$ \\
\hline $\begin{array}{l}\text { Lot } 1 \\
\text { Témoin }\end{array}$ & 225,8 & 40,1 & 14,3 & $2,4_{4}$ & 6,37 & 0,33 & 13,2 & 2,4 & 5,85 & 0,28 \\
\hline $\begin{array}{c}\text { Lot } 2 \\
1 \text { traite } \\
\text { par jour }\end{array}$ & 214,0 & 38,6 & 14,3 & 2,7 & 6,70 & 0,58 & 13,2 & 2,4 & 6,18 & 0,37 \\
\hline $\begin{array}{c}\text { Lot } 3 \\
\text { Pas de traite } \\
\text { le dimanche soir }\end{array}$ & 196,9 & 43,6 & 12,3 & 2,3 & 6,30 & 0,47 & 11,9 & 2,0 & 6,13 & 0,36 \\
\hline $\begin{array}{l}\text { Lot } 4 \\
\text { Aucune traite } \\
\text { le dimanche }\end{array}$ & 172,0 & 45,2 & 10,9 & 3,1 & 6,37 & 0,50 & 10,3 & 2,9 & 6,00 & 0,24 \\
\hline $\mathrm{F}$ & $\begin{array}{l}3,72 \\
*\end{array}$ & & $\begin{array}{l}4,53 \\
* *\end{array}$ & & $\begin{array}{c}1,69 \\
\text { NS }\end{array}$ & & $\begin{array}{c}3,79 \\
*\end{array}$ & & $\begin{array}{l}2,61 \\
\text { NS }\end{array}$ & \\
\hline
\end{tabular}

(1) 21 semaines $=147$ jours.

* différence significative au niveau $5 \%$

** différence significative au niveau $1 \%$

NS non significative 
2. Au cours de la lactation.

Si l'on examine l'évolution des moyennes journalières au fur et à mesure du déroulement de l'expérience (fig. I) on constate que les écarts maximums par rapport au lot témoin se "creusent " au cours du premier mois et parfois même au cours de la première semaine (lot 4). Ces écarts ont tendance à s'atténuer légèrement par la suite.

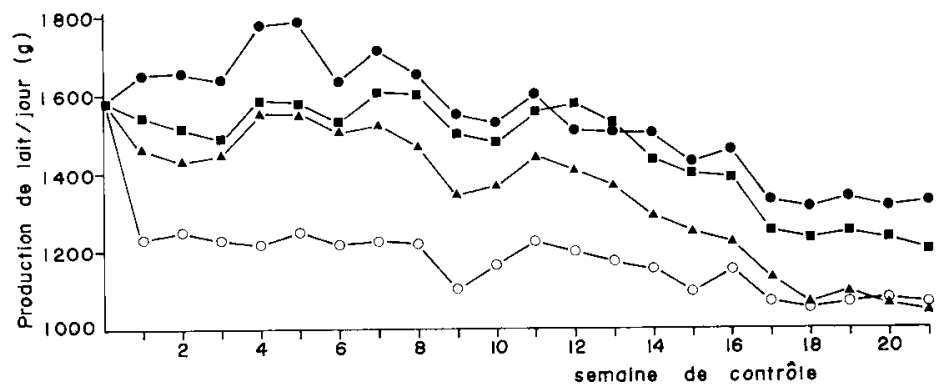

FIG. I. - Courbe de lactation des 4 groupes de brebis soumises à 4 fréquences de traite différentes

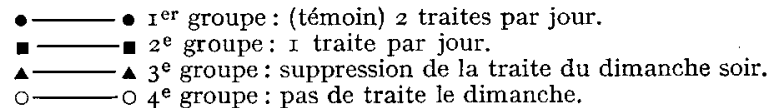

3. Au cours de la semaine (fig. 2 et tab1. 2)

Sur le groupe I témoin, la production du soir est légèrement plus faible que celle du matin (environ $25 \mathrm{~g}$ en moins, soit $3 \mathrm{p}$. Ioo, et ceci bien que les intervalles entre traites soient strictement égaux.)
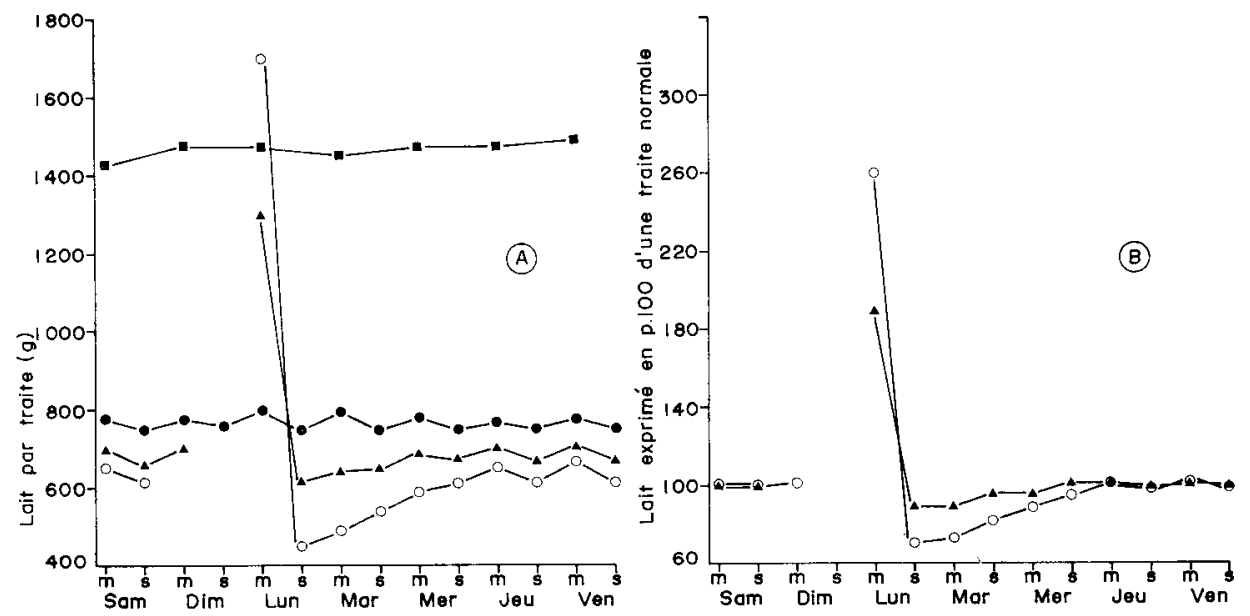

FIG. 2. - Evolution de la production laitière au cours de la semaine

A : en valeurs absolues.

$\mathrm{B}$ : en valeurs relatives par rapport à une traite normale.

Légende identique à celle de la figure I

Le total journalier de ce lot est à peine supérieur à l'unique et très régulière récolte quotidienne $\mathrm{du}$ groupe 2 . Lors de la suppression de la traite du dimanche soir (groupe 3) la quantité de lait recueillie le lundi matin est de I 300 g, ce qui représente 189 p. roo de celle obtenue un matin normal. Elle descend en dessous de la normale à partir du lundi soir (62I g) et ceci jusqu'au mercredi. 


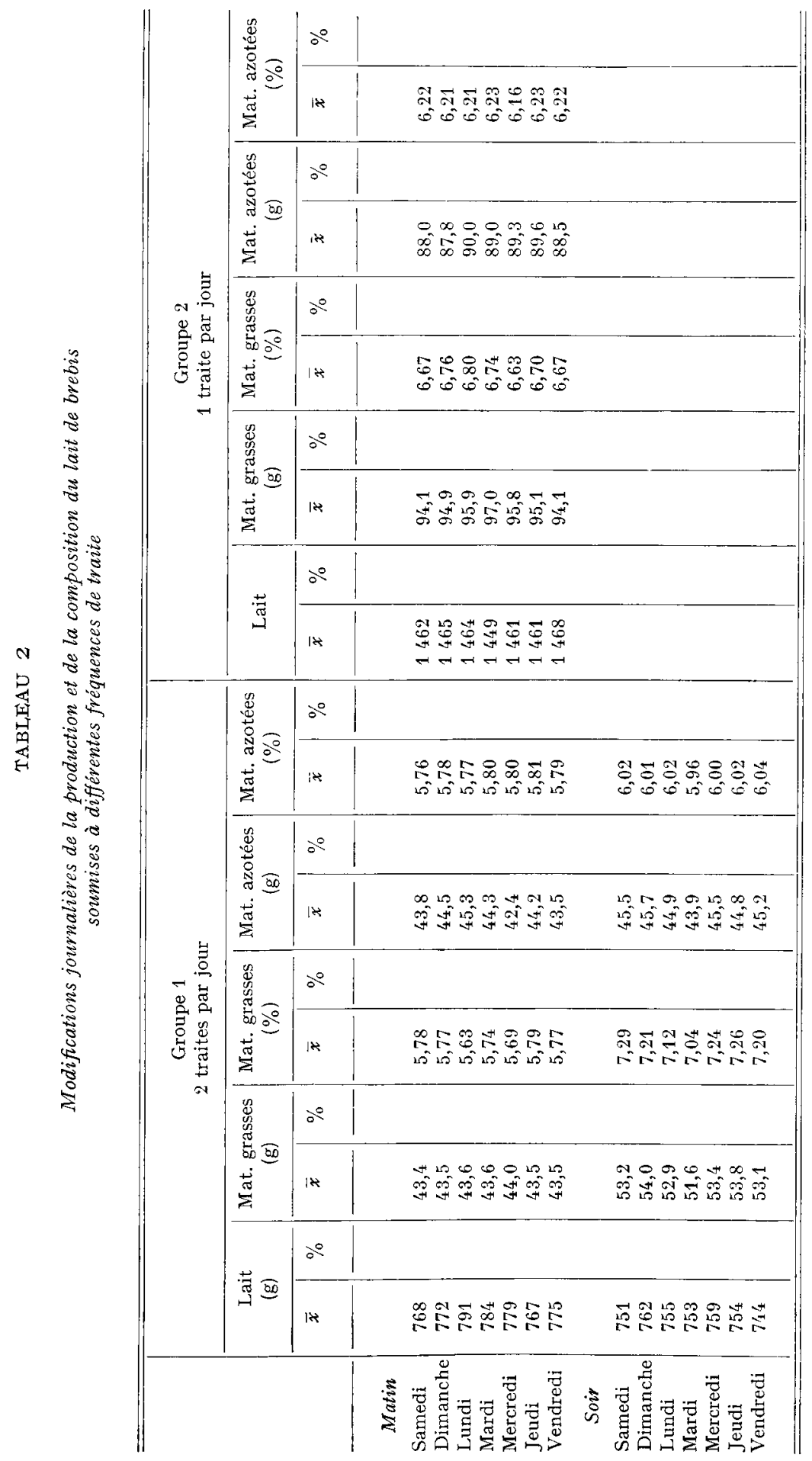




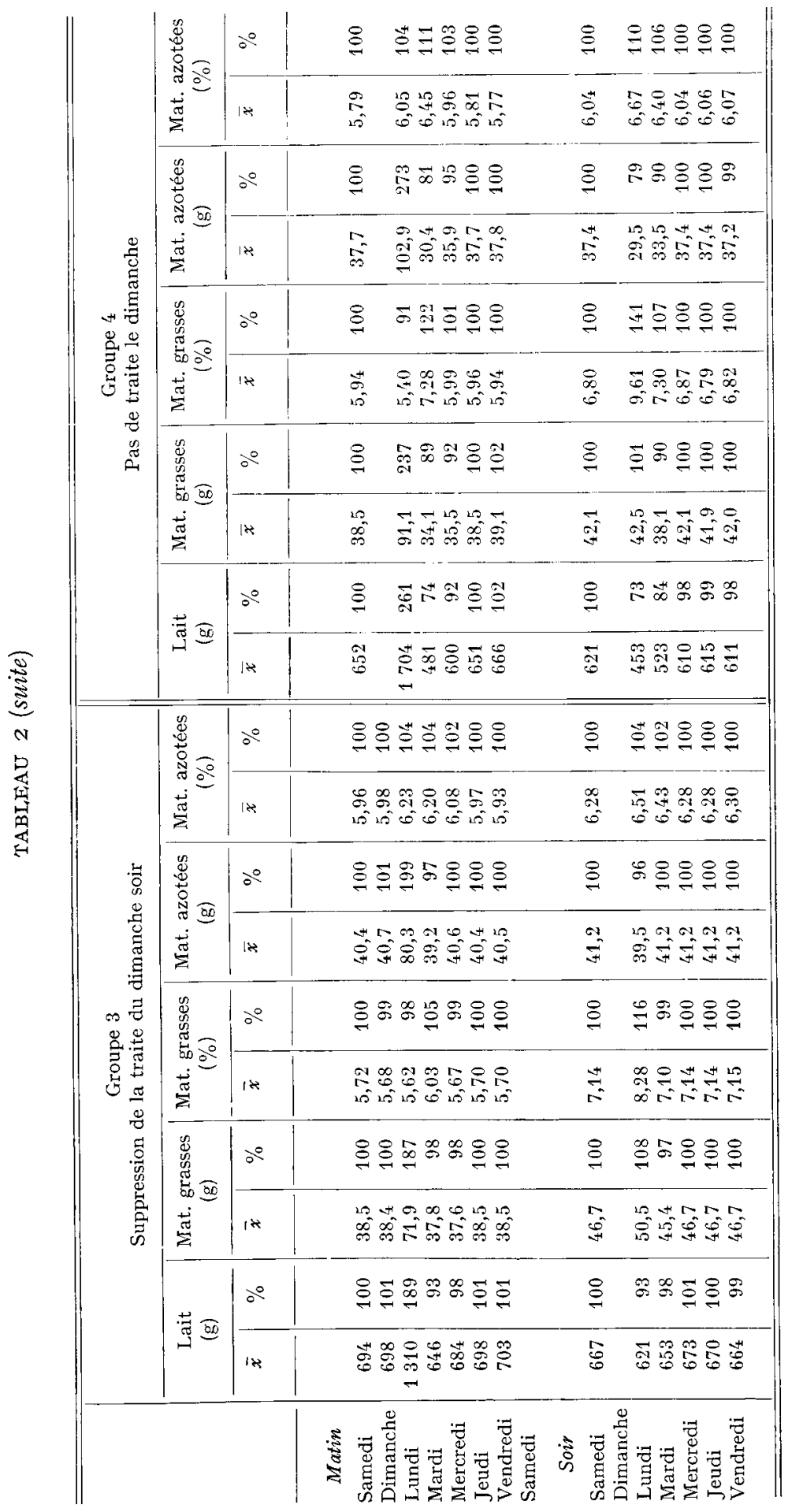


Dans le cas où l'on ne trait plus du tout le dimanche, les écarts sont encore plus importants : r $704 \mathrm{~g}$ le lundi à 6 heures et seulement 453 le même jour à I 8 heures (respectivement $26 \mathrm{I}$ p. Ioo et $73 \mathrm{p}$. Ioo d'une traite ordinaire) ; ces perturbations se prolongent cette fois jusqu'au jeudi matin.

\section{B. - Composition du lait}

I. Bilan des 147 jours (tabl. I).

Les taux butyreux et azoté moyens ne diffèrent pas d'un groupe à l'autre, bien que la pratique d'une seule traite par jour ait tendance à fournir un lait légèrement plus riche. On observe pourtant des effets] significatifs en ce qui concerne les quantités totales de matières grasses et de matières azotées produites : celles-ci sont en effet identiques pour les 2 premiers groupes ( $\mathrm{I}$ et 2 ) alors qu'elles sont nettement plus basses pour les 2 autres (3 et 4 ) (de 1'ordre de 24 et I 4 p. Ioo pour les graisses et de 22 p. Ioo et ro p. Ioo pour les protéines).
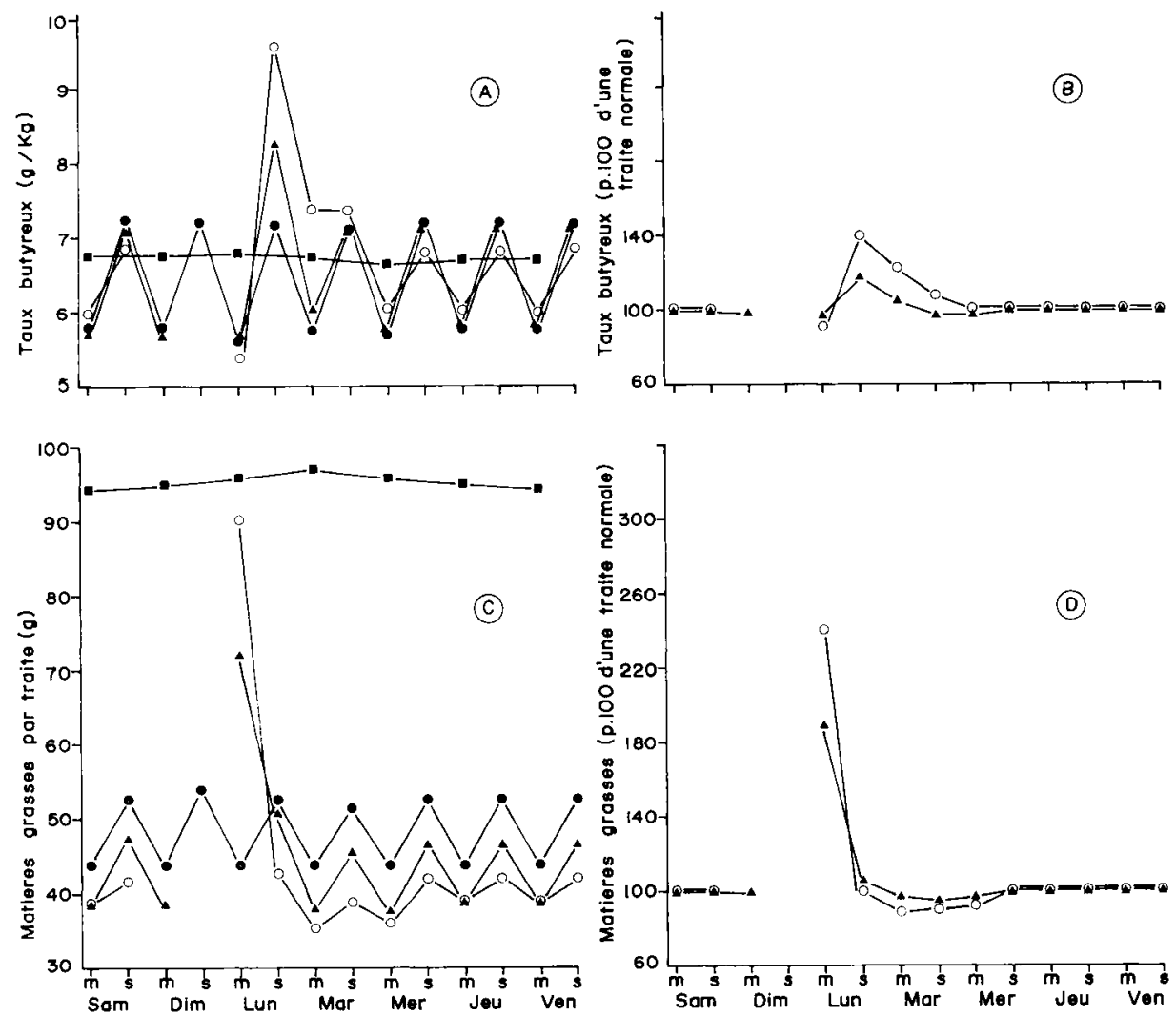

FIG. 3. - Évolution de la sécrétion des matières grasses du lait au cours de la semaine.

A. - taux butyreux en valeurs absolues.

B. - taux butyreux en valeurs relatives par rapport à une traite normale.

C. - matières grasses totales en valeurs absolues.

D. - matières grasses totales en valeurs relatives par rapport à une traite normale.

- - i er groupe (témoin) : 2 traites par jour.

- $2^{\mathrm{e}}$ groupe : I traite par jour.

$\Delta 3^{\mathrm{e}}$ groupe : suppression de la traite du dimanche soir.

$\circ-04^{\mathrm{e}}$ groupe : pas de traite le dimanche. 
2. Au cours de la semaine (tabl. 2, fig. 3 et 4 ).

a) Taux butyreux et matières grasses (fig. $3 a$ et $3 b$ ).

Le groupe I présente les oscillations physiologiques régulières entre matin et soir déjà observées précédemment (CASU, I965).

Au contraire sur le lot 2, il n'y a pratiquement pas de variations d'un jour à l'autre.
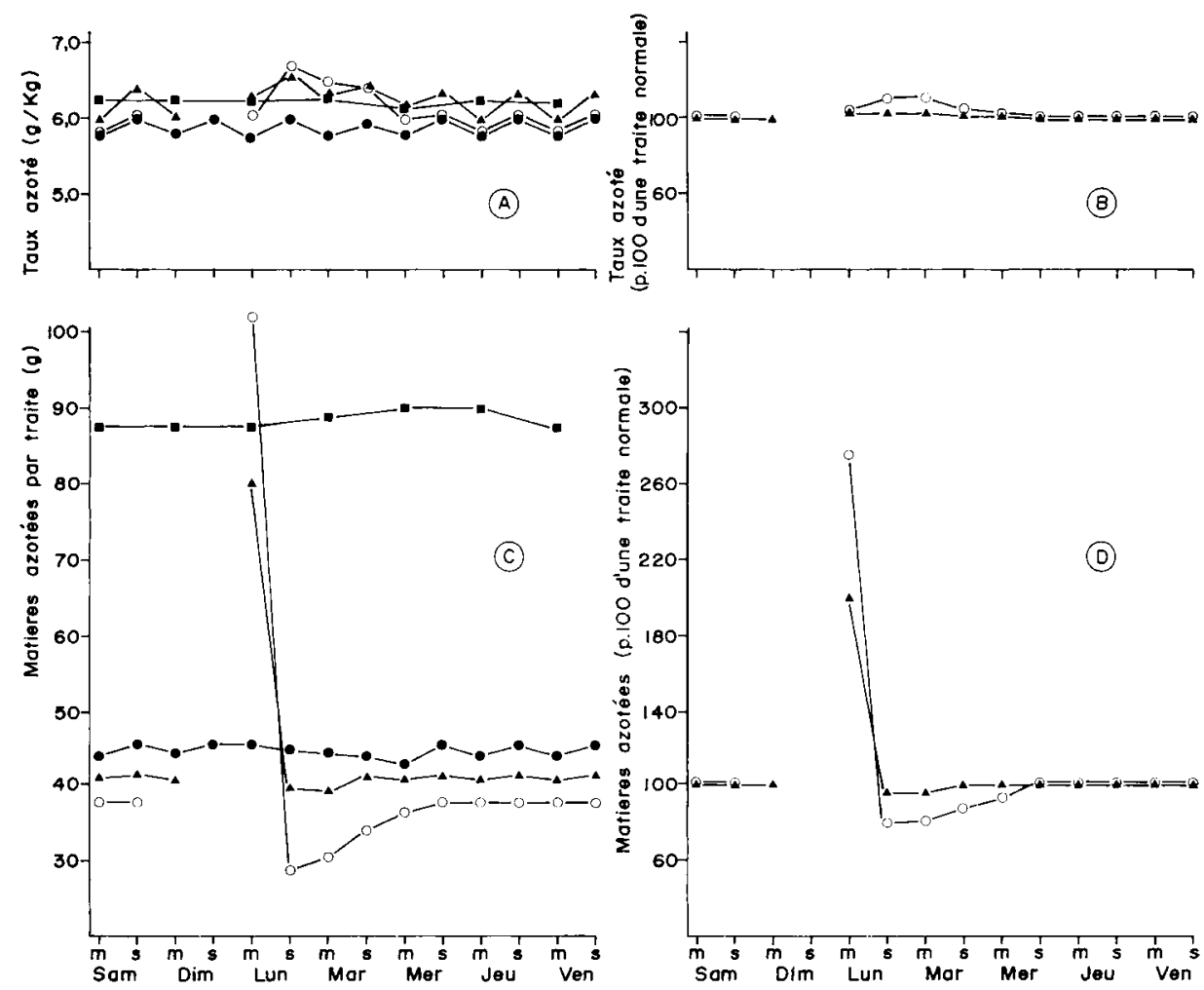

FIG. 4. - Evolution de la sécrétion des matières azotées du lait au cours de la semaine

A. - taux azoté en valeurs absolues.

13. - taux azoté en valeur relative par rapport à une traite normale.

C. - matières azotées totales en valeur absolue.

D. - matières azotées totales en valeurs relatives par rapport à une traite normale.

- - I er groupe (témoin) 2 traites par jour.

- groupe $x$ traite par jour.

_- $\_3^{\mathbf{e}}$ groupe suppression de la traite du dimanche soir.

$\mathrm{O}-\mathrm{O} 4^{\mathrm{e}}$ groupe pas de traite le dimanche.

Après l'omission d'une ou deux traites le dimanche, il est surprenant de constater un taux butyreux pratiquement normal le lundi matin alors qu'il est extrêmement élevé le lundi soir (en particulier pour le lot 4).

Des valeurs plus fortes qu'à l'accoutumée se prolongent jusqu'au mardi soir inclus. Elles se répercutent sur les matières grasses totales dont les quantités recueillies sont identiques aux autres jours de la semaine malgré la production laitière plus faible pendant cette période (fig. $3 c$ et $3 d$ ). 


\section{b) Taux azotés et protéines (fig. 4).}

Bien que pour ces constituants, les modifications de la composition du lait soient perçues encore le mercredi matin, on peut dire d'une façon générale que l'omission d'une ou deux traites consécutives provoque des effets moins spectaculaires sur le taux azoté que sur le taux butyreux (en particulier en ce qui concerne le pic du lundi soir).

\section{C. - Etat sanitaire de la mamelle}

L,es contrôles périodiques effectués à l'aide du CMT mettent en évidence, pour les groupes 3 et 4 , une augmentation du nombre de leucocytes du lait uniquement pendant les 4 premières traites qui suivent la rétention lactée.

Aucun cas de mammites cliniques sur ces deux lots n'a été relevé au cours de l'expérience.

\section{DISCUSSION ETT CONCLUSION}

La suppression des deux traites du dimanche entraîne une forte diminution de la production laitière en I47 jours $(23,9$ p. roo) et altère de façon notable, quantitativement et qualitativement, la sécrétion du lait jusqu'au mercredi. Il est vrai que dans un tel cas, 1'intervalle de 36 heures entre le samedi soir et le lundi matin est trop éloigné des $\mathrm{r} 6$ heures considérées comme la limite supérieure compatible avec une activité normale du tissu mammaire. (ELLIOT, I959).

Il paraît donc difficile d'envisager une telle solution dans les conditions actuelles de l'élevage méditerranéen.

En revanche, les perturbations provoquées par l'omission de la traite du dimanche soir sont beaucoup moins importantes puisque la perte n'est alors que de I2,7 p. Ioo. Ces chiffres sont sensiblement plus faibles que ceux observés par l'un de nous (LABUSSIÈrRE, I972) dans la même espèce sur la race Préalpes $d u$ Sud.

Cette aptitude de la brebis Sarde à supporter de grands intervalles de traite est sans doute due aux particularités de stockage considérable des parties citernales de la glande mammaire.

Ainsi, et bien que les renseignements recueillis au cours de ce premier essai ne puissent conduire à des conclusions définitives (compte tenu du nombre limité de sujets contrôlés), il est extrêmement intéressant de souligner la faible réduction de production laitière consécutive à la pratique d'une seule traite quotidienne (environ 5,2 p. IOO) et le peu de modifications de la composition du lait qui en résulte.

Précisons en particulier que les animaux soumis à ce traitement fournissent encore en moyenne $2 I_{4} \mathrm{~kg}$ en I 47 jours (soit I,46 kg/jour) certaines brebis atteignant même des maxima de $285 \mathrm{~kg}$ (soit I,94 kg/jour). Bien que dans un tel cas la quantité totale de matières grasses et de matières azotées se soit révélée égale à celle du lot témoin, nous devons assortir ces résultats de sérieuses réserves puisque la formation des lots au début de l'essai ne tenait pas compte de la composition du lait. 
C'est pourquoi, avant de diffuser une telle méthode, il importe d'étendre cette expérimentation à d'autres troupeaux, afin de confirmer ces premières données extrêmement encourageantes pour l'avenir de l'élevage ovin laitier.

Reçu pour publication en décembre 1971.

\section{SUMMARY}

\section{FIRST RESUL,TS CONCERNING THE OMITTING}

\section{OF ONE OR TWO MILKINGS PER WEEK IN SARDINIAN EWES}

Fourty-eight Sardinian ewes were subdivided into 4 homogeneous groups after an experimental period of $\mathrm{r}_{5}$ days during which all the animals were milked twice a day.

The further treatments applied to these 4 groups were the following :

group $\mathrm{r}: 2$ milkings per day at $6 \mathrm{a} . \mathrm{m}$. and $6 \mathrm{p}$. m. (controls)

- 2: I milking per day at 6 a.m.

- $\quad 3: 2$ milkings per day from Monday till Saturday and only one milking on Sunday at 6 a.m.

- 4:2 milkings per day from Monday till Saturday and omitting of the two milkings on Sunday.

The results obtained tend to prove that Sardinian ewes are able to support large milking intervals. This is probably due to the high storage capacity of the cisternal parts of their mammary glands. Compared to the control group, the milk yields of groups 2,3 and 4 were reduced by $5.2 \mathrm{p}$. I0o, $12.7 \mathrm{p}$. Ioo and $23.9 \mathrm{p}$. Ioo respectively (table I).

The butterfat and protein contents did not significantly vary from one group to another. However, the total amount of fat and protein produced during the experimental periori (2 I weeks), were lower when the milking intervals increased (especially for group 4, table I).

As already noted for cows, the secretion and composition of the milk were much affected until Wednesday. Therefore, the farms which intend to adopt one of these techniques, cannot be subjected to milk controls before Thursday (fig. 2, 3 and 4 , table 2).

None of the 4 groups of ewes examined showed clinical mastitis during the course of the experiment. However, in groups 3 and 4 , an increase of the number of leucocytes in the milk was observed for 4 days following the milk retention (Test C. M. T.).

\section{RÉFÉRENCES BIBLIOGRAPHIQUES}

CASU S. r965. Richercha sulla composizione del latte di pecora di razza sarda. Alenni fattore di variazione della percentuale di grasso nel latte. Science e tecnica lattiero casearia. Anno XVI, $\mathrm{n}^{\circ} 4$.

Claesson O., Hansson A., Gustafsson N., Brannang E., I959. Studies on monozygons cattle twins. XVI. Once a day milking compared with twice a day milking. Acta Agriculturae Scandinavica. 9, $\mathbf{r}, 38-58$.

Claesson O., I962. A 6 I/2 day week for cows. Dairy farmer. 9, 36-38.

ElıIOT G. M. I959. The direct effect of milk accumulation in the udder of the dairy cow upon milk secretion rate. Dairy Sci. Abstr. 21, Io, 435-439.

Labussière J., Richard Ph., I965. La traite mécanique. Aspects anatomiques, physiologiques et technologiques. Mise au point bibliographique. Amm. Zootech., 14, I, 63-I 26.

Labussiere J., Coindet J., I 968 Effets de la suppression de la traite du dimanche soir chez les bovins de race Française Frisonne Pie Noire. Ann. Zootech. 17, 3, 231-234.

LABUSSière J., 1972. Effets de la suppression de la traite du dimanche soir chez les brebis laitières de race Préalpes $d u$ Sud. Ann. Zootech. (à paraître). 